\title{
Who will determine the educational needs of our trainees?
}

\author{
Ara Asadur Vaporciyan, MD
}

I read the editorial written by Dr Akins with great interest. In it, he makes an appeal to our society that American Board of Thoracic Surgery (ABTS) certification, which recognizes graduates as competent to practice both cardiac and general thoracic surgery, is no longer appropriate. He proposes that we should allow certification in cardiac or general thoracic surgery and presents a number of cogent arguments supporting his premise. The arguments proposed center on 2 areas. The first is focused on the justification most commonly cited supporting the ABTS's position, that the data derived from our society's workforce studies demonstrate that a large number of graduates practice both disciplines. Dr Akins's contention is that these data are insufficient justification. The second set of arguments all focus on the benefit we would derive educationally by allowing our trainees and our training programs to focus on one or the other discipline. These include elements such as the ability to decrease total training time, to make the field more attractive to applicants who have a single focus, to adhere to duty hour restrictions, and to improve trainee education by allowing trainees to focus their attention on one discipline or the other.

I will not attempt to dispute Dr Akins's later arguments regarding the educational benefits of a dual pathway credentialing system for those trainees who desire a career in a single discipline. Clearly, there are some trainees who are certain that they will practice only 1 discipline and would benefit from a streamlined educational experience. These benefits include reduced training time as well as a more focused educational experience. Indeed, the availability of separate credentialing might well attract more of these individuals to our profession. Program directors face a constant struggle to adhere to duty hour restrictions. A focused educational experience would allow program directors greater flexibility to adjust schedules and meet those requirements. Finally, our training environment, the exposure to cases, the complexity of these cases, and our learners themselves are radically different from those 20 (or even 10) years ago. All these statements regarding the educational benefits of 2 focused credentialing options are accurate and cannot

From the Department of Thoracic and Cardiovascular Surgery, University of Texas M.D. Anderson Cancer Center, Houston, Tex.

Disclosures: Authors have nothing to disclose with regard to commercial support.

Received for publication May 4, 2012; revisions received May 16, 2012; accepted for publication June 8, 2012; available ahead of print July 3, 2012.

Address for reprints: Ara Asadur Vaporciyan, MD, University of Texas M.D. Anderson Cancer Center, Thoracic and Cardiovascular Surgery, 1515 Holcombe Blvd, Box 445, Houston, TX 77030 (E-mail: avaporci@mdanderson.org).

J Thorac Cardiovasc Surg 2012;144:532-3

$0022-5223 / \$ 36.00$

Copyright (c) 2012 by The American Association for Thoracic Surgery

http://dx.doi.org/10.1016/j.jtcvs.2012.06.010 be contested. These arguments hinge on Akins's first argument, however, that our workforce study findings are not sufficient justification to maintain our current credentialing requirements. If that argument is not upheld, then the additional arguments focused on the benefits of educating trainees in a dual credentialing system are no longer relevant. This key argument, the validity of using workforce data to drive the goals of our curriculum, is an issue of curriculum design, specifically the needs assessment.

A curriculum's focus is based on the needs of all stakeholders who will benefit from the final product of that curriculum. When training cardiothoracic surgeons, the stakeholders include patients, trainees, professional society members, hospitals, and payers (including the government and the taxpayers). These stakeholders decide on the needs of our curriculum. Once these needs have been established, the rest of the curriculum design process is focused on meeting them. The ABTS, whose primary mission is to protect the public, is well positioned to speak for patient stakeholders. It also represents our professional society because its membership is drawn from the ranks of this society. Although it certainly does not represent all stakeholders, it does represent the significant majority.

The ABTS has used workforce data as a major element of the needs assessment. I would contend that the use of these data as a needs assessment for our curriculum is the best available form of a needs assessment. If not practice pattern surveys, then what other data should we use to make curricular decisions? Practice pattern surveys are the single best source of data for a needs assessment, because they include multiple stakeholders rather than the views of selected experts. These data represent the real world of cardiothoracic surgery, and as such they identify what patients in the general community desire as well as what our professional society membership feels should be provided. One can certainly argue that patient care might be improved if we were to focus on only 1 discipline, but the reality is that the majority $(>70 \%)$ of our graduates engage in both disciplines. If this is the best representation of the current status of cardiothoracic surgery, then our educational systems must meet that reality. Educational systems should meet the needs of a society, not impose a new paradigm.

If the majority of stakeholders believe that the current approach is flawed, however, then a new paradigm may be necessary. This is the only justification for splitting into dual credentialing. Like Dr Akins, I recognize the educational (and quality of care) improvements achieved by focusing on one aspect of cardiothoracic surgery. There must, however, be a consensus before such a change. In addition, there are many issues that would need to be 
reconciled if such a move were contemplated. Certainly, dual certification would maximize the educational experience of the $30 \%$ of graduates focused on one branch of our profession. But, we would now require at least 2 (a cardiac and a thoracic credentialing) and maybe even 3 (a cardiac, a thoracic, and a combined credentialing) separate certifications.

If we have only 2 certificates, then $70 \%$ of our graduates would have to complete multiple certifications to reach their goal. Alternatively, if we have a combined certificate to serve those $70 \%$, what would be included in their scope of practice? This need to choose or potentially restrict a trainee's practice may be just as large (or even larger) a drain on our applicant pool. The fact is that the current need to train and be credentialed in both disciplines has not deterred the flood of medical students applying to the integrated 6-year programs.

Another related argument is that initial career paths and job opportunities are highly erratic. Although some trainees may desire a practice focused on 1 discipline, the reality is that if they are to secure a job, they may need to work in other areas, if only to make themselves more attractive to initial employers. Alternatively, an initial job with a broad clinical focus may later shift into a focused practice in one discipline. This may come from internal pressures, such as the individuals' own personal choice, or from external pressures, such as changing practice patterns. For these reasons, it may be advantageous to provide alternative pathways during maintenance of certification rather than at initial Board certification. By the time an individual is due for maintenance of certification, he or she will have an established practice pattern and could be certified in the relevant discipline or disciplines only. This was, in fact, Dr Akins's assertion in his recently published editorial ${ }^{1}$ regarding recertification.
Finally, if we do develop separate pathways and their associated certification, how will we prevent activity outside one's discipline? This is probably the hardest issue with which to contend. If the focus is to be on patient safety, as Dr Akins asserts, then we will need to enforce that graduates of 1 discipline do not expand their practice into the other discipline. An example of just how difficult this might be comes from the fact that general surgeons still perform general thoracic procedures, despite the existence of the ABTS since 1948. This is not an argument for or against separation into dual credentialing. The decision to have dual credentialing should come from our educational needs, as derived from all stakeholders. All curriculums must exist, however, within the reality of the environment in which they were created. The reality of health care in America is that market forces drive clinical care. How will we enforce this separation when the entrenched culture is one that allows cardiothoracic surgeons to practice both disciplines?

In summary, I support Dr Akins's argument that dual credentialing would improve the ability to teach those trainees focused on one discipline. The needs driving our curriculum and the realities of health care delivery in America, however, support the goals of our curriculum as it is currently established. In the end, I do not deny that separation has benefits, but rather I contend that there would be significant (although perhaps not insurmountable) challenges that would need to be overcome both in the design of our curriculum and in the delivery of health care to generate such a change. I applaud Dr Akins for opening discourse in this subject. The discussion must continue, because its resolution requires input from a wide variety of stakeholders.

\section{Reference}

1. Akins CW. The ethical dilemma of thoracic surgical recertification. J Thorac Cardiovasc Surg. 2012;143:521-2. 